\title{
Power spectra from spotted accretion discs
}

\author{
Tomáš Pecháček, ${ }^{1,2}$ Michal Dovčiak ${ }^{2}$ and Vladimír Karas ${ }^{2}$ \\ ${ }^{1}$ Charles University, Faculty of Mathematics and Physics, Prague, Czech Republic \\ ${ }^{2}$ Astronomical Institute, Academy of Sciences, Prague, Czech Republic \\ email: pechacek_t@seznam.cz
}

\begin{abstract}
Some aspects of power-spectral densities (PSD) of active galactic nuclei are similar to those of galactic black hole X-ray binary systems (McHardy et al. 2005). The signal originates near a black hole and its modulation by general-relativistic effects should be taken into account (Życki \& Nedźwiecki 2005). We modified the previous calculations of these effects, assuming a model of spots which occur on the disc surface and decay with a certain lifetime.
\end{abstract}

Keywords. Accretion disks, black hole physics, methods: analytical, X-rays: general

\section{Introduction}

Let us consider an infinitesimal surface element, $r \mathrm{~d} r \mathrm{~d} \phi$, residing a thin accretion disc near a non-rotating black hole. This element of area is assumed to be orbiting together with the disc material with Keplerian orbital frequency, $\Omega \equiv \Omega(r)$. The redshift factor $g\left(\phi, r, \theta_{\mathrm{o}}\right)$ (which defines the change of photon energy $\nu_{\mathrm{o}} / \nu_{\mathrm{s}}$ with an observer inclination $\left.\theta_{\mathrm{o}}\right)$ and the total flux received by a detector $F\left(\phi, r, \theta_{\mathrm{o}}\right)$ can be approximated by (Pecháček et al. 2005, 2006)

$$
\begin{gathered}
g=\frac{\sqrt{r(r-3)}}{r+\sin \phi \sin \theta_{\mathrm{o}} \sqrt{r-2+4\left(1+\cos \phi \sin \theta_{\mathrm{o}}\right)^{-1}}}, \\
F \equiv \frac{f}{I}=g^{4}\left[1+\frac{1}{r} \frac{1-\sin \theta_{\mathrm{o}} \cos \phi}{1+\sin \theta_{\mathrm{o}} \cos \phi}\right] \cos \theta_{\mathrm{o}} .
\end{gathered}
$$

The exact calculation of the time delay in the curved spacetime leads to elliptic integrals (e.g. Cadež \& Kostić 2005). Using the power series expansion method several simple approximative formulae can be found. According to Beloborodov (2002) the light bending in Schwarzschild spacetime can be with high accuracy described by the relation $1-\cos \alpha=$ $(1-\cos \psi)(1-u)$ where $u=2 / r, \alpha$ is the angle of emission with respect to the radial direction in the local static frame and $\psi$ is the angle measured in the orbital plane of the photon from the emission point to the direction of the observer. By expanding in $(1-\cos \alpha) /(1-u)$ up to the second order, Poutanen \& Beloborodov (2006) give

$$
u \Delta t=(1-\cos \psi)\left(1+\frac{1}{8} u(1-\cos \psi)[1+(1-\cos \psi)(1 / 3-u / 14)]\right),
$$

where $\Delta t$ is the delay between signal emitted from some $\psi$ and signal emitted radially toward a distant observer from the same initial distance $u$ as the first signal.

Collecting all terms depending linearly on $u$ we found a slightly different expression,

$$
u \Delta t=(1-\cos \psi)-u\left[\frac{1}{2}(1-\cos \psi)+\ln (1-(1-\cos \psi) / 2)\right] .
$$

Accuracy of different approximations is assessed in figure 1. 

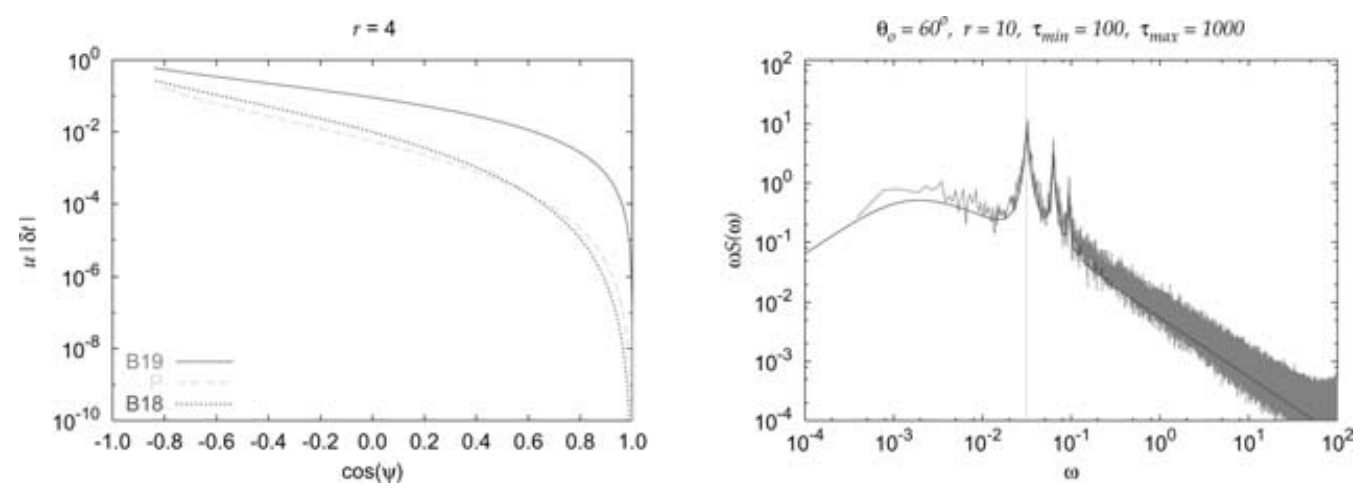

Figure 1. Left: Comparison of three different approximations for the time delay. The difference of the approximation and exact numerical solution is plotted. The solid line "B18" refers to (1.3), "B19" corresponds to $(1-\cos \psi)$ (i.e. the first term of (1.3)) and "P" stands for (1.4). Right: Power spectrum from the spot model calculated for a narrow ring $r=10$, observer's inclinations $\theta_{\mathrm{o}}=60^{\circ}$, and life-time intervals $\left\langle\tau_{\min }, \tau_{\max }\right\rangle$. The red curve is a result of direct numerical simulation. Dashed blue curve is an approximation calculated from formula (2.1) assuming probability density function $\rho(\tau) \propto 1 / \tau$. The vertical lines give the Keplerian orbital frequency $\Omega(r)$ for the corresponding radius.

\section{Effects of decaying spots on the disc}

As a simple model of the X-ray flux variability we consider a stochastic process consisting of independent exponentially decaying events, with uniformly distributed ignition times. After averaging we find PSD in the form

$$
S(\omega)=n \sum_{k=-\infty}^{\infty}\left|c_{k}\right|^{2} \int_{\tau_{\min }}^{\tau_{\max }} \rho(\tau) \frac{\tau^{2}}{1+\tau^{2}(\omega-k \Omega)^{2}} \mathrm{~d} \tau,
$$

where $\Omega$ and $c_{k}$ are the frequency and Fourier series coefficients of $F(t), n$ is a mean rate of events and $\rho(\tau)$ is the probability density function of the random variable $\tau$. See Pecháček et al. (2006) for details.

\section{Results \& Conclusions}

We have developed approximative analytical formulae useful to describe relativistic effects acting on photons that originate from spots on a geometrically thin accretion disc near the Schwarzschild black hole. We used these formulae to derive the theoretical variability power spectra and compared their shapes with the corresponding power spectra generated by a numerical routine.

We thank the Czech Science Foundation (ref. 205/03/H144) and the Grant Agency of the Academy of Sciences (ref. 300030510) for support.

\section{References}

Beloborodov, A. M. 2002, ApJ 586, L85

Čadež, A. \& Kostić, U. 2005, Phys. Rev. D 72, 104024

McHardy, I. M., Gunn, K. F., Uttley, P. \& Goad, M. R. 2005, MNRAS 359, 1469

Misner, C. W., Thorne, K. S. \& Wheeler, J. A. 1973, Gravitation (Freeman: San Francisco)

Pecháček, T., Dovčiak, M., Karas, V. \& Matt, G. 2005, A\&A 441, 855

Pecháček, T., Dovčiak, M., Karas, V. \& Matt, G. 2005, AN 327, 957

Poutanen, J. \& Beloborodov, A. M. 2002, MNRAS 373, 836

Życki, P. T. \& Nedźwiecki, A. 2005, MNRAS 359, 308 